\title{
Economic analysis of use of counterfeit drugs: health impairment risk of counterfeit phosphodiesterase type 5 inhibitor taken as an example
}

\author{
Minoru Sugita • Michiko Miyakawa
}

Received: 28 October 2009/Accepted: 5 January 2010/Published online: 9 February 2010

(C) The Japanese Society for Hygiene 2010

\begin{abstract}
Objectives The size of the market for counterfeit drugs throughout the world is considerable. Many cases of health impairment due to counterfeits have been reported. The market share of counterfeits in drug markets in developed countries is smaller than that in developing countries. However, the size of the market for counterfeits of phosphodiesterase type 5 inhibitors (PDE5Is) used as antierectile-dysfunction drugs is not small. The purpose of the present study was to analyze the health impairment risk of taking the counterfeit PDE5Is and the convenience of obtaining the counterfeits in Japan, using an economic methodology in order to work out countermeasures for reducing the health impairment risk.

Methods Information was obtained by interviewing employees of pharmaceutical and chemical corporations in Japan.

Results and discussion The size of the market for counterfeit PDE5Is in Japan was recently estimated to be about 2.5 times larger than that of genuine PDE5Is. The price of the counterfeits in their market is reported to be nearly equal to that of the genuine PDE5Is. An outbreak of severe hypoglycemia among users of counterfeit PDE5Is containing an antidiabetic drug in Singapore was reported in 2008, and seven patients remained comatose as a result of
\end{abstract}

\section{Sugita $(\square)$}

Department of Environmental and Occupational Health,

Toho University School of Medicine,

5-21-16 Omori-nishi, Ota-ku,

Tokyo 143-8540, Japan

e-mail: sugita@med.toho-u.ac.jp

M. Miyakawa

Faculty of Humanity and Environment,

Hosei University, Tokyo, Japan prolonged neuroglycopenia. Four of them subsequently died, so the health impairment risk due to counterfeit PDE5Is should not be ignored. In order to obtain a genuine PDE5I in Japan, a patient must be examined and have a prescription written at a medical institution, and buy it at a dispensing pharmacy. Focusing on the health impairment risk due to counterfeit PDE5Is and the convenience of obtaining the counterfeits in Japan, we analyzed the effects on the prices and quantities of PDE5Is in the market from demand and supply curves, using an economic methodology. From the analysis, it was shown that the health impairment risk due to the counterfeits is underestimated in the market in Japan. Physicians should warn their patients not to buy counterfeit PDE5Is, and when they write a prescription for purchasing genuine PDE5Is, should inform their patients of the severe health problems that occurred in Singapore.

Conclusions The present economic analysis indicates that the health impairment risk due to counterfeit PDE5Is is underestimated in the market in Japan. Clarification of the underestimation of the severe health impairment risk due to counterfeits is important.

Keywords Counterfeit - PDE5 inhibitor · Health impairment risk - Convenience of acquisition .

Economic analysis

\section{Introduction}

The history of counterfeits is as old as the history of commodities. Scarce commodities of high quality are supplied in the market and consumers evaluate the commodities highly. If consumers become dissatisfied with the high price and/or difficulty in obtaining the commodities, suppliers of 
counterfeits appear. Counterfeit goods look like the genuine article but the quality is not the same. When the price of a product rises due to monopoly and/or taxation, the supply of counterfeits often grows. Counterfeits of branded goods and drugs in developing countries and counterfeit cigarettes in European countries are some examples. The counterfeits of Tamiflu ${ }^{\circledR}$ (oseltamivir; Roche) and the anti-erectiledysfunction drugs phosphodiesterase type 5 inhibitors (PDE5Is) are examples that show the difficulty and inconvenience of obtaining the genuine drugs. Counterfeits of these pharmaceuticals are distributed widely.

The World Health Organization (WHO) defines a counterfeit medicine as one which is deliberately and fraudulently mislabeled with respect to identity and/or source [1]. Counterfeiting can apply to both branded and generic products, and counterfeit products may include products with the correct ingredients or with the wrong ingredients, without active ingredients, with insufficient active ingredients, or with fake packaging. What makes a drug/medicine counterfeit is the deliberate or intentional (criminal) nature of the mislabeling of the product. WHO established the International Medical Products AntiCounterfeiting Taskforce (IMPACT) as a countermeasure against counterfeit drugs [2].

WHO presents the situation of counterfeit distribution in the world as follows [3]. Counterfeiting is greatest in those regions where regulatory and enforcement systems for medicines are weakest. Thus, in most industrialized countries with effective regulatory systems and market control, the incidence of counterfeit medicines is extremely lowperhaps less than $1 \%$ of market value according to the estimates of the countries concerned. But in many African countries, and in parts of Asia and Latin America, a much higher percentage of the medicines on sale may be counterfeit. In many countries of the former Soviet Union, counterfeit medicines are estimated to exceed $20 \%$ of market value. Medicines purchased over the Internet from illegal sites that conceal their physical address have been found to be counterfeit in more than $50 \%$ of cases. The testimony given by Peter J. Pitts, the Director of the Centre for Medicines in the Public Interest (CMPI) noted that counterfeit business will generate 75,000 million dollars in revenues for owners in 2010, a 92\% increase from 2005 [4]. A strong trend is observed towards increasingly professional PDE5I counterfeits [5]. The International Intellectual Property Institute (IIPI) described counterfeit goods as not only an intellectual property and trade problem, but also as an unrecognized global public health problem with particular consequences in the area of injury, mortality, and morbidity [6]. An outbreak of severe hypoglycemia among users of counterfeit PDE5Is containing an antidiabetic drug in Singapore was reported in 2008; 150 patients were admitted to hospitals and seven patients remained comatose as a result of prolonged neuroglycopenia, and four subsequently died [7].

No cases of health impairment due to taking counterfeit PDE5Is have been reported in Japan. Some reasons for this are that counterfeit medicines are not taken under the supervision of physicians and/or pharmacists, no system for collecting information on adverse counterfeit drug reactions has been put in place by pharmaceutical corporations, and consumers of counterfeits are reluctant to report adverse drug reactions due to counterfeits [8]. In Japan, however, counterfeits should not be ignored as social and economic problems. Countermeasures against health impairment risks and the infringement of intellectual property rights by the counterfeit PDE5Is have been worked out by pharmaceutical corporations [9-14]. Risk analysis of health impairments due to counterfeit PDE5Is in Japan has been investigated [8].

However, economic analysis for market of counterfeit goods including pharmaceuticals has not been studied at all. The purpose of the present study was to conduct an analysis of the health impairment risk due to counterfeit PDE5Is and the convenience of obtaining the counterfeits in Japan, using an economic methodology, making it possible to work out countermeasures for reducing the health impairment risk.

Illegal goods are divided into counterfeit and imitation goods. Counterfeit goods look like the genuine goods, having the same shape, color, and embossing. Imitations are defined as illegal products that do not look like the genuine goods, but by their name or claim strongly suggest that the quality of the imitations is almost equal to that of the genuine goods, in spite of having risks of health impairment and low quality. It has been reported that the difference between the health impairment risks due to counterfeit and imitation PDE5Is was not large [5]. Recently, information was obtained that cheap illegal imitation PDE5Is made in India are being distributed in Japan [15]. The illegal imitation PDE5Is are generic drugs from India. Drug patents are not stipulated strictly in the law in India. Therefore, drugs patented in developed countries can be produced as generic drugs in India without the payment of a patent fee. When such generic drugs are imported from India to developed countries, the generic drugs are illegal in the developed countries. Health impairments caused by taking the generic PDE5Is have not been reported in India. In the present study, the illegal imitation PDE5Is are not included, because a probable estimate of the imitation PDE5Is cannot be determined.

In the present study, a genuine PDE5I means a PDE5I that is not generic or sold over-the-counter (OTC). An OTC PDE5I means a PDE5I which can be obtained without a prescription at pharmacies, while a genuine PDE5I can be obtained only with a prescription. 


\section{Materials and methods}

Information was obtained by interviewing chemical engineers and pharmaceutical chemists at five pharmaceutical corporations and one chemical corporation and a professional employee in a section for countermeasures against counterfeits in a pharmaceutical corporation in Japan. The interview was conducted in the year from 2006 to 2009 . The publication of the names of the employees interviewed and their corporations is not consented to. They showed us articles in print and Internet URLs for information on the genuine and counterfeit PDE5Is and the prices and quantities of the PDE5Is distributed as rough estimates.

\section{Results and discussion}

Information on the prices and quantities of genuine, OTC, generic, counterfeit, and imitation PDE5Is was obtained in the form of rough estimates from an interview with a professional employee of a section for countermeasures against counterfeits in a pharmaceutical corporation. The data are shown in Table 1. The reason for the use of the rough estimates is that precise values cannot be obtained objectively. The mean acquisition price is indicated as the value when a consumer purchases ten PDE5I tablets. If many PDE5I tablets are purchased with a prescription, the mean value varies considerably, while the value without a prescription does not vary. However, consumers seldom purchase many PDE5I tablets for individual use. Therefore, the variation in the number of tablets obtained is not significant. In Table 1, the values shown for OTC and generic PDE5Is are assumed values. The mean price of the counterfeit PDE5I is nearly equal to that of the genuine PDE5I, while the value of the imitation is cheaper than that of the genuine PDE5I. The market size of the counterfeit PDE5Is in Japan was recently estimated to be about 25,000 million yen, 2.5 times larger than that of the genuine PDE5Is [16,
17]. The number of counterfeit PDE5Is confiscated by Japanese customs has slightly increased recently [18] because the printed fake trade name of the genuine PDE5I on the surface of the box of the counterfeits is conspicuous. Therefore, the quantity of counterfeits distributed was estimated to have slightly decreased recently in Japan. A counterfeit medicine is deliberately and fraudulently mislabeled with respect to identity and/or source [1]. If the price of a counterfeit medicine is much cheaper than that of the genuine medicine, consumers of the counterfeit may suspect that the medicine is a counterfeit. Therefore, suppliers of counterfeits set the price to be nearly equal to that of the genuine product. The prices of OTC and OTC generic PDE5Is will be cheaper than that of the genuine PDE5Is in the future, and the quantities distributed will increase. The OTC genuine PDE5Is will be sold in the future by major corporations that sell the genuine PDE5Is at present, while the generics will be sold by medium and small-sized corporations. The brand image of the genuine PDE5Is supplied by major corporations will be considered more valuable than that of the generics supplied by medium and small-sized corporations. Therefore, the quantity of generics distributed, especially those supplied by medium and small-sized corporations, will increase only slightly in the future, despite cheaper prices. Quantities of the generics distributed and their prices will take a long time to rise and fall.

The values shown in Table 1 were obtained by rough estimations because precise values cannot be obtained objectively. In the present study, demand and supply curves derived from the values in Table 1 were drawn for an economic analysis.

In Japan, three brands of genuine PDE5Is are sold at monopoly prices under the patents. In the present study, the hypothetical situation that generic PDE5Is will be sold in the future after the patents expire is discussed using an economic methodology. In the present study, $p, q, D$, and $S$ indicate price, quantity, and demand and supply curves.

Table 1 Prices and relative consumption of genuine, OTC genuine, generic, OTC generic, counterfeit, and imitation PDE5Is

\begin{tabular}{llllll}
\hline & Prescription & Price & & $\begin{array}{l}\text { Relative consumption } \\
\text { and its increase } \\
\text { or decrease }\end{array}$ \\
\cline { 3 - 5 } & & $\begin{array}{l}\text { Medical and } \\
\text { prescription fees }\end{array}$ & $\begin{array}{l}\text { Purchase price } \\
\text { for ten tablets }\end{array}$ & $\begin{array}{l}\text { Mean acquisition } \\
\text { price for one tablet }\end{array}$ & 1 \\
\hline Genuine & + & $¥ 3,000$ & $¥ 12,000 / 10 \mathrm{~T}$ & $¥ 1,500 / \mathrm{T}$ & $3(\nearrow)$ \\
OTC genuine & - & $¥ 0$ & $¥ 12,000 / 10 \mathrm{~T}$ & $¥ 1,200 / \mathrm{T}$ & $1(\nearrow)$ \\
Generic & + & $¥ 3,000$ & $¥ 12,000 / 10 \mathrm{~T} \times 0.7$ & $¥ 1,140 / \mathrm{T}(\searrow)$ & $4(\nearrow)$ \\
OTC generic & - & $¥ 0$ & $¥ 12,000 / 10 \mathrm{~T} \times 0.7$ & $¥ 840 / \mathrm{T}(\searrow)$ & $2.5 \searrow$ \\
Counterfeit & - & $¥ 0$ & $¥ 15,000 / 10 \mathrm{~T}$ & $¥ 1,500 / \mathrm{T}$ & $1 \nearrow$ \\
Imitation & - & $¥ 0$ & $¥ 15,000 / 10 \mathrm{~T} \times 0.8$ & $¥ 1,200 / \mathrm{T}$ & \\
\hline
\end{tabular}

Arrows current increase or decrease, (arrows): increase or decrease in the future

PDE5Is phosphodiesterase type 5 inhibitors, OTC over-the-counter 
Fig. 1 Prices and quantities expressed by demand and supply curves of genuine and generic phosphodiesterase type 5 inhibitors (PDE5Is)
Fig. 2 Prices and quantities expressed by demand and supply curves of genuine and over-the-counter (OTC) PDE5Is

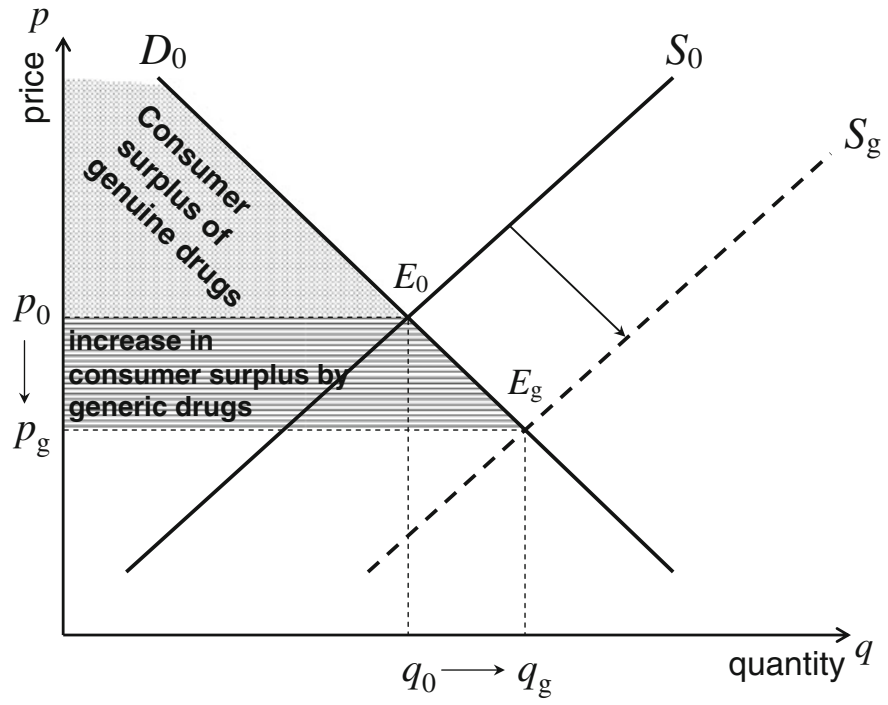

$D$ : demand curve, $S$ : supply curve, 0: genuine PDE5I, g: generic PDE5I (future)

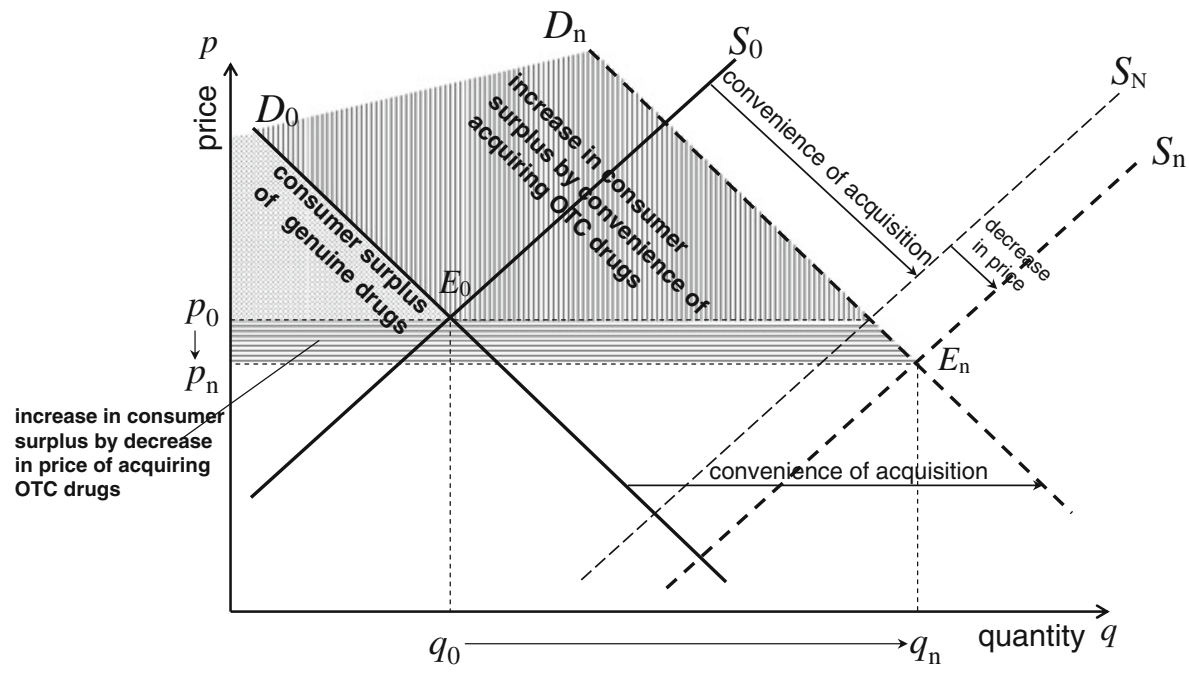

$D$ : demand curve, $S$ : supply curve, 0: genuine PDE5I, n: OTC PDE5I (future),

$\mathrm{N}$ : OTC PDE5I whose price of acquisition is the same as that of genuine PDE5I (supposition)
Figure 1 shows the influence on price and quantity of demand and supply due to the generic PDE5Is, using the demand and supply curves. The subscripts in Fig. 1, "0" and "g", indicate genuine and generic PDE5Is. This kind of figure is used in many textbooks of economics [19-21]. Figure 1 shows that generics result in a fall in price and a rise in quantity of demand and supply. The Figures in the present study indicate steady states a long time after generic and OTC PDE5Is go on sale, because steady states are assumed in classical methods of economic analysis. The lateral stripes in Fig. 1 show an increase in consumer surplus by generics. The consumer surplus is the difference between the total amount that consumers are willing and able to pay for a good or service, indicated by the demand curve, and the total amount that they actually do pay. Patents last for some time, and generic PDE5Is will not be sold in the near future. Therefore, Fig. 1 shows a hypothetical scenario.

In Japan, in order to obtain a genuine PDE5I, a patient must be examined by a physician and have a prescription written at a medical institution, and purchase the genuine PDE5I at a dispensing pharmacy upon submitting the prescription. In the present study, the hypothetical situation that an individual can purchase OTC PDE5Is without a prescription is investigated using an economic methodology. Figure 2 shows the influence on price and quantity of demand and supply by the OTC PDE5Is using demand and supply curves. The subscripts in Fig. 2, "0", "n", and "N" 
Fig. 3 Prices and quantities expressed by demand and supply curves of genuine and counterfeit PDE5Is

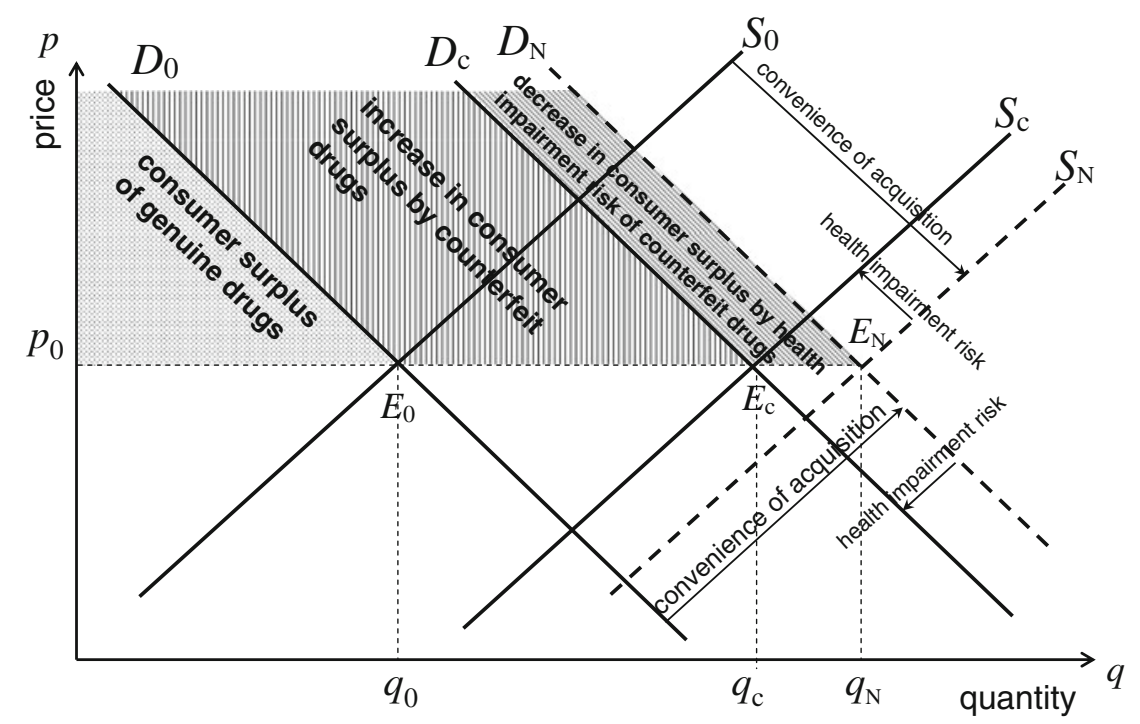

$D$ : demand curve, $S$ : supply curve, 0: genuine PDE5I, c: counterfeit PDE5I, $\mathrm{N}$ : OTC PDE5I whose price of acquisition is the same as that of genuine PDE5I (supposition) indicate genuine PDE5Is, OTC PDE5Is, and hypothetical OTC PDE5Is whose price is nearly equal to that of the genuine PDE5I. The price of the OTC drug does not include medical and prescription fees. Therefore, the price of the OTC drug is cheaper than that of the genuine drug. Figure 2 shows that the OTC PDE5Is raise the quantity of the demand and supply due to the lower price as well as the convenience of obtaining the OTC PDE5Is. When the movement of the supply curve $S$ in Fig. 2 is broken down into movements due to a fall in price and those due to the convenience of obtaining the OTC PDE5Is, the movement due to the convenience of obtaining the OTC PDE5Is goes from $S_{0}$ to $S_{\mathrm{N}}$ and the movement due to the fall in price goes from $S_{\mathrm{N}}$ to $S_{\mathrm{n}}$. The vertical and lateral stripes in Fig. 2 show increases in consumer surpluses by convenience of obtaining the OTC PDE5Is and by fall in price. In Fig. 2, the increase in consumer surplus by convenience of obtaining the OTC PDE5Is is much larger than that by the fall in price. OTC PDE5Is will not go on the market in Japan in the near future because the Ministry of Health, Labour and Welfare considers the health impairment risk due to the coadministration of a PDE5I and drugs involving nitric oxide donors, organic nitrites, or nitrates to be too great. Therefore, Fig. 2 is a hypothetical scenario.

From an Internet survey, a pharmaceutical corporation obtained information indicating that a major reason that consumers purchase counterfeit PDE5Is instead of genuine PDE5Is is the convenience of obtaining PDE5Is without having to be examined at a medical institution. This information has not been published.
Figure 3 indicates the influence on price and quantity of demand and supply by counterfeit PDE5Is using demand and supply curves. The subscripts in Fig. 3, "0", "c", and "N" indicate genuine PDE5Is, counterfeit PDE5Is, and hypothetical OTC PDE5Is whose price is nearly equal to that of the genuine PDE5I. If a counterfeit PDE5I whose quality is nearly equal to that of the genuine PDE5I is supplied, its supply curve should correspond fairly closely to the curve of the hypothetical OTC PDE5I whose price is nearly equal to that of the genuine PDE5I. Therefore, the supply curves of the two can be regarded as being almost the same. However, there are risks of health impairment and low quality in counterfeit PDE5Is. Some counterfeit PDE5Is with quality as high as that of genuine PDE5Is were found [5]. However, before chemical analysis is conducted for a distributed counterfeit, the magnitude of its risk cannot be known. Therefore, there are no counterfeits distributed without risk.

Most counterfeit PDE5Is are distributed by use of the Internet. The prices of the counterfeit PDE5Is were investigated using the Internet, and it was revealed that the mean price of the counterfeit PDE5Is was nearly equal to that of the genuine PDE5Is (Table 1). In Fig. 3, the demand and supply curves $D_{\mathrm{c}}$ and $S_{\mathrm{c}}$ of the counterfeit PDE5I are shown to move in parallel from the demand and supply curves $D_{0}$ and $S_{0}$ of the genuine PDE5I and/or from the demand and supply curves $D_{\mathrm{N}}$ and $S_{\mathrm{N}}$ of the hypothetical OTC PDE5I whose price is nearly equal to that of the genuine PDE5I. The intersection point of the demand and supply curves $D_{\mathrm{c}}$ and $S_{\mathrm{c}}$ of the counterfeit PDE5I is $E_{\mathrm{c}}$, 
$\left(q_{\mathrm{c}}, p_{\mathrm{c}}\right)$ in Fig. 3. The movement direction of the demand and supply curves from $D_{0}$ and $S_{0}$ of the genuine PDE5I to $D_{\text {c }}$ and $S_{\text {c }}$ of the counterfeit PDE5I for price and quantity determination is shown as a large movement to the right from $D_{0}$ and $S_{0}$ to $D_{\mathrm{N}}$ and $S_{\mathrm{N}}$ due to the convenience of obtaining the counterfeits and a slight movement to the left from $D_{\mathrm{N}}$ and $S_{\mathrm{N}}$ to $D_{\mathrm{c}}$ and $S_{\mathrm{c}}$ due to the risks of health impairment and low quality of the counterfeit PDE5I in the plane of price and quantity in Fig. 3. In any case, the quantity of the demand or the supply of the counterfeit PDE5Is is much greater than that of the genuine PDE5Is and is slightly less than that of the hypothetical OTC PDE5Is whose price is nearly equal to that of genuine PDE5Is. The size of the market for the counterfeit PDE5Is in Japan was recently estimated to be about 25,000 million yen, 2.5 times larger than that of the genuine PDE5Is [16, 17]. Figure 3 shows that the quantity $q_{\mathrm{c}}$ of the supply and/ or the demand of the counterfeit PDE5Is is much greater than that $q_{0}$ of the genuine PDE5Is.

If the importance of the health impairment risk due to use of the counterfeit PDE5Is is evaluated appropriately in the market for the counterfeits, the price of the counterfeits should be much cheaper than that of the genuine PDE5Is and the quantity of counterfeits distributed should be less than the current quantity of the counterfeits. The major reason that consumers purchase counterfeits is probably the convenience of obtaining them without having to be examined at medical institutions. In the case where the quality and quantity of casualties and damage may be great when the risk is known, but the probability of the risk becoming known is low, a person usually prefers to take the risk when he/she thinks that the advantage of taking the risk is greater than the risk itself [22]. This is human nature. Suppliers of counterfeits know about the low quality of the

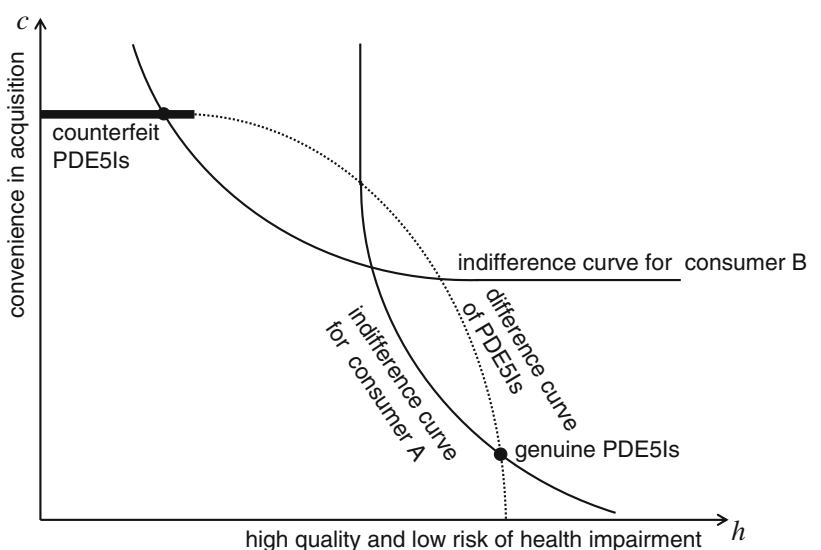

Fig. 4 Theoretical difference curve of PDE5Is on a plane of high quality and convenience of acquisition in a differentiation market of genuine and counterfeit PDE5Is and indifference curves for consumers counterfeits, while consumers of the counterfeits can seldom know the true risk. This is surely asymmetric information [23, 24].

A net increase in the consumer surplus by convenience of obtaining the counterfeits is the sum of an apparent increase in the consumer surplus by convenience (vertical stripes in Fig. 3) and the decrease in the consumer surplus by the risk due to the counterfeits (diagonal stripes in Fig. 3). Figure 3 shows that the magnitude of the decrease in the consumer surplus by the risk due to counterfeits (diagonal stripes) is much smaller than those of the apparent (vertical stripes) and net increases in consumer surplus by the convenience of the counterfeits. The increase in the consumer surplus by convenience is enlarged inappropriately because the consumers of counterfeits choose inappropriately to obtain the counterfeits conveniently. If the consumers of counterfeits could fully understand the importance of the health impairment risk due to the counterfeits, the vertical stripes portion in Fig. 3 would decrease, and the diagonal stripes portion in Fig. 3 would increase. The consumer surplus by the counterfeits is an indicator that both the price and the quantity are considered together, using the demand and supply curves for convenience of obtaining counterfeits and their risk. From the viewpoint of the consumer surplus, an overestimate of the convenience of obtaining the counterfeits and an underestimate of their risk in the market for the counterfeits were clearly found in the present study. In any case, as a social problem, the health impairment risk due to the use of counterfeit PDE5Is should not be ignored.

Great increases in the consumer surplus by convenience of obtaining the counterfeit PDE5Is (Fig. 3) and OTC PDE5Is (Fig. 2) are shown in an economic analysis of the price and quantity of the demand and supply in the market, while a decrease in consumer surplus by health impairment risk due to the counterfeits (Fig. 3) and an increase in the consumer surplus by the price fall of the generic PDE5Is are not great (Fig. 1). This shows the use of an economic methodology for detecting the importance of the convenience of obtaining counterfeit PDE5Is in the market to be significant.

In marketing, product differentiation is the process of distinguishing a product from competitors' products to make it more attractive to a target differentiation market. Differentiation is a source of competitive advantage. A differentiation market of genuine and counterfeit PDE5Is should be discussed. Counterfeit PDE5Is are supplied conveniently without a prescription for consumers by suppliers of counterfeits, while genuine PDE5Is can be obtained only by prescription. There are nonnegligible risks of health impairment and low quality in counterfeit PDE5Is, while the quality of genuine PDE5Is is high. A theoretical difference curve of PDE5Is on a plane of high 
quality $(h)$ and convenience of acquisition $(c)$ in a differentiation market of genuine and counterfeit PDE5Is, derived assuming that production costs without research and development costs are constant [25], and indifference curves of consumers, are shown in Fig. 4. The quality of counterfeit PDE5Is is distributed very widely [5]. Therefore, the bold line in Fig. 4 shows the quality and convenience of acquisition of counterfeits. The dotted part of the theoretical difference curve in Fig. 4 may not exist, because convenience means with or without a prescription. The indifference curve is the expression in a graph of the utility function for a consumer [19-21, 25], where the horizontal axis measures high quality and low risk of health impairment and the vertical axis measures convenience of acquisition of PDE5Is. A consumer can refer equally to each point on the indifference curve as rendering the same level of utility (satisfaction) for him/herself. In Fig. 4, consumer A prefers the quality of PDE5Is to convenience of acquisition and obtains a genuine PDE5I, while consumer B prefers convenience to quality and purchases a counterfeit PDE5I. The size of the market for counterfeit PDE5Is is much greater than that of genuine PDE5Is. This means that there are many PDE5Is consumers who regard convenience of acquisition as important.

As an effective countermeasure against the health impairment risk due to counterfeits, physicians should inform their patients about the health impairment risk and warn them not to purchase counterfeit PDE5Is. The patient should be informed that taking counterfeit PDE5Is means taking the risk of health impairment e.g., severe hypoglycemia, which occurred among users of counterfeit PDE5Is in Singapore; 150 patients were admitted to hospitals and seven patients remained comatose as a result of prolonged neuroglycopenia, and four subsequently died [7]. From an Internet survey, a pharmaceutical corporation obtained information that many consumers of counterfeit PDE5Is would change to genuine PDE5Is if they were informed of the health impairment risk by physicians. This information has not been published. It is important to be aware of the danger of underestimating the severe health impairment risk due to counterfeits.

A pharmaceutical corporation obtained information from an Internet survey that a major reason that consumers of counterfeit PDE5Is purchase the counterfeits instead of the genuine PDE5I is the convenience of obtaining PDE5Is without having to undergo examination at a medical institution. It is shown in Fig. 2 that the increase in the consumer surplus due to the convenience of obtaining the OTC PDE5Is is much larger than that due to low price. A pharmaceutical corporation has obtained information that selling PDE5Is as OTC drugs is being discussed in the European Union (EU). In order to exclude counterfeit PDE5Is from the market, it may be effective to offer
PDE5Is as OTC drugs, taking into consideration the convenience of obtaining them.

Homo economicus, a term used as an approximation or model of Homo sapiens who acts to obtain the highest possible wellbeing for oneself given the available information about opportunities and other constraints, both natural and institutional, on one's ability to achieve predetermined goals, was assumed in classical economics. In behavioral economics, however, it has been pointed out that the above is seldom true. In behavioral economic studies of addictions such as smoking, it has been mentioned that the rate of time preference of smokers is higher than that of nonsmokers and the measure of risk aversion is lower [26]. The risk aversion measure of consumers of counterfeit PDE5Is is probably low. As an effective countermeasure against the health impairment risk due to counterfeits, physicians should inform patients about the possibility of their having a low aversion measure of the health impairment risk and encourage them to purchase genuine PDE5Is by prescription.

Good research is conducted on past events, but this is not the case for research on future events. It is impossible to verify future events in studies. No cases of health impairment due to taking PDE5I counterfeits have been reported in Japan. Some reasons for this may be that counterfeit medicines are not taken under the supervision of physicians and/or pharmacists, no system for collecting information on adverse counterfeit drug reactions has been developed by pharmaceutical corporations, and consumers of counterfeits are reluctant to report adverse counterfeit drug reactions, for various reasons [8]. The reported probability of health impairment cases due to taking counterfeit PDE5Is is underestimated in Japan. In the present study, research on health impairment cases due to taking counterfeit PDE5Is was conducted based on uncertain information, using study methods that cannot be analyzed precisely. However, when we take social problems into consideration, the significance of the study is not small.

For the area of the present study, it was very difficult to obtain precise, quantified, and objective information. Therefore, the scales of the ordinates and the abscissas in Figs. 1, 2, 3, and 4 are not known, so the linearity or curvature of the demand and supply curves drawn as lines in Figs. 1, 2, and 3 is not known. It is possible, however, to make a rough comparison of the magnitudes of the consumer surpluses in Figs. 1, 2, and 3, e.g., the findings that the magnitude of the increase in consumer surplus by convenience of obtaining the counterfeits is much greater that of the decrease in consumer surplus by the health impairment risk due to the counterfeits are roughly known. If more information in the area of the present study can be obtained, knowledge will increase. If notable and nonnegligible health impairment risks are found, appropriate research for the risks can be conducted and appropriate countermeasures against 
the risks can be worked out, even if it is very difficult to obtain precise, quantified, and objective information in the area. We should not consider that research in which the obtaining of precise, quantified, and objective information is difficult is unscientific and that such research is not worth conducting. As a social problem, the health impairment risk due to the use of counterfeit PDE5Is should not be ignored. Risks due to counterfeit PDE5Is involve not only the health impairment risk investigated in the present study but also risks to public order and security.

\section{Conclusions}

The price of PDE5I counterfeits in the market is reported to be nearly equal to that of the genuine PDE5Is. The counterfeit PDE5Is in Japan has recently been estimated to be about 2.5 times larger than that of the genuine PDE5Is. Focusing on the health impairment risk due to counterfeit PDE5Is and the convenience of obtaining the counterfeits in Japan, we analyzed the effects on the price and quantity from demand and supply curves, using an economic methodology. The analysis showed that the health impairment risk due to the counterfeits is underestimated and convenience of obtaining them is overestimated by consumers in the market. As an effective countermeasure against the health impairment risk, physicians should warn their patients not to buy the counterfeits and should inform them of the severe health impairments that occurred in Singapore. It is important to be aware of the underestimation of the severe health impairment risk due to counterfeits.

Acknowledgments This article is a modified version of a paper presented at the 79th Annual Meeting of the Japan Society for Hygiene, March 2009, and the 20th Annual Meeting of the Japan Society for Sexual Medicine, September 2009, Tokyo, Japan. We express deep appreciation to Professor Emeritus Tadao Miyakawa of Hitotsubashi University for his support. This study was not supported by the pharmaceutical corporations that develop or sell the genuine PDE5Is.

\section{References}

1. World Health Organization. How does WHO define a counterfeit drug (medicine)? 2005. http://www.who.int/medicines/services/ counterfeit/faqs/05/en/.

2. World Health Organization. International Medical Products AntiCounterfeiting Taskforce (IMPACT). 2006. http://www.who.int/ medicines/services/counterfeit/faqs/count_q-a/en/.

3. World Health Organization. Counterfeit medicines: Key facts. 2009. http://www.who.int/medicines/services/counterfeit/CfeitsFact SheetJuly09.pdf.

4. Pitts PJ. 21st Century International Drug Terrorism. Testimony by CMPI Director, Peter J. Pitts, to the Government Reform Committee Subcommittee on Criminal Justice, Drug Policy and Human Resources. 2005. http://special.pacificresearch.org/pub/ sab/health/2005/21_Century_Counterfeiting_Testimony.pdf.
5. Venhuis BJ, Barends DM, Zwaagstra ME, de Kaste D. Recent developments in counterfeits and imitations of Viagra, Cialis and Levitra: a 2005-2006 update. RIVM Report 370030001. 2007. http://www.rivm.nl/bibliotheek/rapporten/370030001.pdf.

6. Forzley M. Counterfeit goods and the public's health and safety. International Intellectual Property Institute (IIPI). 2003. http:// www.iipi.org/reports/Counterfeit_Goods.pdf.

7. Kao SL, Chan CL, Tan B, Lim CC, Dalan R, Gardner D, et al. An unusual outbreak of hypoglycemia. N Engl J Med. 2009; 360:734-6.

8. Sugita M. Risk analysis for health impairment due to the use of counterfeit drugs in Japan using phosphodiesterase type 5 inhibitor (PDE5I) as an example. Nohon Seikino Gakkai-zasshi (Jpn J Sex Med). 2008;23:299-309 (in Japanese with English abstract).

9. Pfizer Japan Inc. Adoption of technology of radio frequency identification (RFID) by Pfizer Japan Inc: for health of patients against counterfeit Viagra: technology against counterfeits suppliers. 2006 (in Japanese).

10. Pfizer Japan Inc. Dangerous counterfeit Viagra. 2008 (in Japanese) http://www.ed-info.net/health.html.

11. Ogasawara N. Forged medicines and its preventive measures. Nihon Hoso Gakkai-shi (J Packag Sci Technol Jpn). 2007; 16:367-75 (in Japanese).

12. Kimura K. Risk by counterfeit medicines which spread globally. Pfizer Forum. 2007;100 (in Japanese) http://www.pfizer.co.jp/ pfizer/healthcare/pfizer_forum/2007/n100.html.

13. Bayer Yakuhin Ltd. Information on an acceptance of an application for prohibiting counterfeits of Levitra ${ }^{\circledR}$, medicine for erectile dysfunction. 2006 (in Japanese) http://byl.bayer.co.jp/ scripts/pages/jp/press_release/press_detail/?file_path $=2006 \% 2$ Fnews2006-12-18.html.

14. Eli Lilly Japan Ltd. Be careful about counterfeits of Cialis ${ }^{\circledR}$ : Additional information about an attention to "ED care support" on a website. 2008 (in Japanese) http://www.lilly.co.jp/CACHE/ news_2008_13.cfm.

15. Sankei Shimbun Co Ltd. Kamagra. Yukan Fuji. 28 Oct 2008 (in Japanese).

16. Yakuji Nippo Ltd. Viagra: Half of drugs imported privately are counterfeits. Yakuji Nippo. 1 Dec 2006 (in Japanese) http:// www.yakuji.co.jp/entry 1744.html.

17. Mainichi Newspapers Co Ltd. Internet retailing of counterfeit "Viagra." Mainichi Newspaper (Osaka version) morning edition, 20 Mar 2007. p. 23 (in Japanese).

18. Nagoya Customs, Japan. Situation of crackdown on violation of Customs Law at Nagoya Customs, Japan in 2008. 2009 (in Japanese) http://www.customs.go.jp/nagoya/content/H20tekihatu_ nagoya.pdf.

19. Cleaver T. Economics: the basics. London: Routledge; 2004.

20. Stiglitz JE, Walsh CE. Economics. 4th ed ed. New York: Norton; 2006.

21. Black J, Hashimzade N, Myles G. A dictionary of economics. 3rd ed ed. Oxford: Oxford University Press; 2009.

22. Tsuchida S. Risk perception and risk communication: The Society for Risk Analysis, Japan. Nihon Eisei Gakkai-zasshi (Jpn J Hyg). 2007;62:325-6 (in Japanese).

23. Arrow KJ. Uncertainty and the welfare economics of medical care. Am Econ Rev. 1963;58:941-73.

24. Akerlof G. The market for lemons: quality uncertainty and the market mechanism. Q J Econ. 1970;84:488-500.

25. Shiozawa S. An introduction to economics. Tokyo, Yuhikaku. 1996 (in Japanese).

26. Goto R, Takahashi Y, Nishimura S, Ida T. A cohort study to examine whether time and risk preference is related to the success of smoking cessation? Addiction. 2009;104:1018-24. 